\title{
Assessing the evidence on aspirin in diabetes mellitus. Gemini or Libra; lumping or splitting; surrogate or hard; low or high; interventionist or nihilist
}

\author{
J.S. Yudkin \\ Department of Medicine, University College London Medical School, Whittington Hospital, London, UK
}

The articles by Drs. Cimminiello and Milani, and by Drs. Ceriello and Motz, review in some detail the literature relating to aspirin therapy in diabetes. Both plead for newer antiplatelet agents of greater efficacy in patients with diabetes, inferring from the available studies that aspirin is not particularly effective in these patients.

A major risk of overinterpreting data from the literature is to look at particular subgroups of just one trial in isolation. Both because of the problem of type 2 error, and as a consequence of multiple comparisons, some very strange things can happen. Thus, the ISIS 2 study [1] showed no benefit from aspirin in patients with myocardial infarction born under the astrological signs of Gemini and Libra. To look at the ISIS 2 study alone as evidence for lack of efficacy of aspirin in diabetic patients may be falling into the same trap.

It is inevitable that small studies are less likely to produce significant results, a phenomenon well recognised as type 2 error. Hence, the power of metaanalysis to combine information from numerous small studies in order to quantify likely benefit. Using this approach, the Antiplatelet Trialists' Collaboration quantified the benefit of antiplatelet agents in preventing vascular events and death in some 47000 patients, of whom nearly $10 \%$ had diabetes [2]. The relative benefit on vascular events was $17 \%$ in the diabetic patients and $22 \%$ in those without diabetes, an insignificant difference. Although the number is lower for diabetic patients than for non-diabetic patients in terms of percentage benefit, the absolute number of events prevented were similar in the two groups (38 per thousand compared with 35 per thousand, respectively) because of the higher background risk in diabetic patients. Furthermore, because the confidence intervals overlap, the presumption must be that benefit is similar in the two groups of patients.
The same concept applies when comparing different antiplatelet agents. In the meta-analysis, some $80 \%$ of patients were given aspirin, so that the accuracy of quantitation of benefit by other agents is of necessity imprecise. It is misleading, then, to extrapolate, on the basis of tiny studies, that one or other newer agent is better (or worse) than aspirin.

Several new drugs which have been assessed are able to block several processes in the cascade of platelet aggregation, and Ceriello and Motz postulate that those operating on mechanisms other than the cyclooxygenase step may be preferred agents in diabetic subjects. There is, however, a major problem with surrogate end points, be they quantified in vitro, ex vitro or in vivo. When we combine our current inability to predict any more than a small component of the apparent randomness of the occurrence of vascular events [3] with the all too obvious fact that too much antiplatelet effect increases the risk of haemorrhagic events (and in particular stroke), it is only with large trials employing hard end points that we will know what we should be doing best to help our patients.

It is, in particular, the balance of benefit and risk that led the Antiplatelet Trialists' Collaboration to their overall conclusion [2]. Overall, the antiplatelet drugs reduced the risk of myocardial infarction by about $30 \%$ and that of cerebral infarction by around $20 \%$, but doubled the incidence of haemorrhagic stroke. As a result, the benefits of antiplatelet drugs were evident in patients at high risk of thrombotic events, those with a history of transient ischaemic attack, stroke, angina or myocardial infarction, usually studied in secondary prevention studies. However, in the results from primary prevention studies, in low risk patients, the increased risk of haemorrhagic stroke outweighed the $20-30 \%$ protection of a thrombotic event that the drug provided.

Another way of approaching the same analysis is to look at the data in terms of background level of 
risk. In the secondary prevention studies, in which antiplatelet drugs showed an overall benefit, the untreated patients had a cardiovascular mortality of 45 per thousand patient years. In the primary prevention studies, where hazards of antiplatelet agents exceeded benefits, the background risk of cardiovascular death was around 4 per thousand patient years. It can be calculated [4] that a middle aged man with diabetes and a moderately raised blood pressure would have a cardiovascular mortality of around 10 per thousand patient years of follow-up, and around twice this level if he were also microalbuminuric. This means that a 50-year-old diabetic man with microalbuminuria, moderately raised blood pressure, or a serum cholesterol concentration over $6.5 \mathrm{mmol} / \mathrm{l}$ would have a cardiovascular risk of the sort of level found in non-diabetic subjects only after myocardial infarction. This means that, on the basis of current evidence, if antiplatelet therapy is warranted by the level of risk, and not by a previous diagnosis of vascular disease, many diabetic patients would also warrant such treatment.

What is needed, and fortunately what is planned, is a major intervention study with aspirin in diabetic patients. The CLIPS study (Critical Leg Ischaemia Prevention Study) is assessing the efficacy of aspirin $100 \mathrm{mg} /$ day, and antioxidant therapy, in a $2 \times 2$ study design in 2100 patients with peripheral vascular disease as evidenced by an ankle to brachial pressure ratio of $\leq 0.85$, with progression of leg ischaemia, vascular events and death as the major end points. But while awaiting results of this important trial, I will continue to recommend aspirin in patients with diabetes who can be calculated to have a risk of cardiovascular death of over 40 per thousand person years of follow-up on the basis of age, gender, smoking, blood pressure, microalbuminuria and lipids. My other bias, admittedly based on a study using surrogate end points [5] is to use $300 \mathrm{mg}$ of enteric coated aspirin daily in these patients [4].

\section{References}

1. ISIS-2 (Second International Study of Infarct Survival) Collaborative Group (1988) Randomised trial of intravenous streptokinase, oral aspirin, both, or neither among 17187 cases of suspected acute myocardial infarction: ISIS2. Lancet II: $349-360$

2. Antiplatelet Trialists' Collaboration (1994) Collaborative overview of randomised trials of antiplatelet therapy. I.Prevention of death, myocardial infarction, and stroke by prolonged antiplatelet therapy in various categories of patients. BMJ 308: 81-106

3. Heller RF, Chinn S, Tunstall Pedoe HD, Rose G (1984) How well can we predict coronary heart disease? Findings in the United Kingdom Heart Disease Prevention Project. BMJ 288: 1409-1411

4. Yudkin JS (1995) Which diabetic patients should be taking aspirin? BMJ 311: 641-642

5. Di Minno G, Silver MJ, Cerbone AM, Morphy S (1986) Trial of repeated low-dose aspirin in diabetes angiopathy. Blood 68: 886-891 